\title{
INTERNADO RURAL EN LA FORMACIÓN MÉDICA
}

\author{
En la Universidad Nacional de San Antonio Abad del Cusco.
}

Santiago Máximo Saco Méndez ${ }^{1}$

\section{RESUMEN}

El Internado Rural Médico contribuye en la mejora de la calidad de los servicios de salud de la comunidad y en la formación integral de los médicos, en la Facultad de Medicina de la Universidad Nacional de San Antonio Abad del Cusco (UNSAAC). Los estudiantes valoran las diversas actividades realizadas en internado, y manifiestan que es una experiencia que contribuye mucho en su formación. PALABRAS CLAVES: Integración docente asistencial, salud comunal, calidad de atención, formación médica.

\section{ABSTRACT}

Rural Internship contributes to improvement of the quality of community health services and the training of physicians at the Faculty of Medicine of The National University of San Antonio Abad of Cusco (UNSAAC). The medical students value the various activities carried out during the Internship, and express that it is an experience that strongly contributes to their training.

KEYWORDS: Integration teaching-assistance, Community health, quality of care, medical education

a Organización Panamericana de la Salud - (OPS), en el Informe Final del grupo reunido en Bahía en 1986, sobre requisitos mínimos para la creación de escuelas de medicina concluyó: "El fin último del sistema de formación de recursos humanos para la salud, no es formar profesionales, sino mejorar la salud de la población.” (1)
En 1978, La Conferencia Internacional sobre la Atención Primaria de la Salud, en Alma Ata, estableció que "uno de los principales objetivos sociales, de los gobiernos, de las organizaciones internacionales y de la comunidad mundial entera en el curso de los próximos decenios debe ser el de que todos los pueblos del mundo alcancen en el año 2000 un nivel de vida social y económicamente productiva. La Atención Primaria de la Salud es la clave para alcanzar esa meta como parte del desarrollo conforme al espíritu de justicia social.” (2)

La Experiencia de la Facultad de Medicina

Humana de la Universidad Nacional de

San Antonio Abad del Cusco

Siguiendo las nuevas tendencias en educación médica y en políticas de salud, y de acuerdo a la Declaración de Alma Ata, la Facultad de Medicina Humana (FMH) de la Universidad Nacional de San Antonio Abad del Cusco, en su proyecto de creación, planteó cambios en la educación médica,

Fotografia 1. Atenciones de emergencia y tópico.

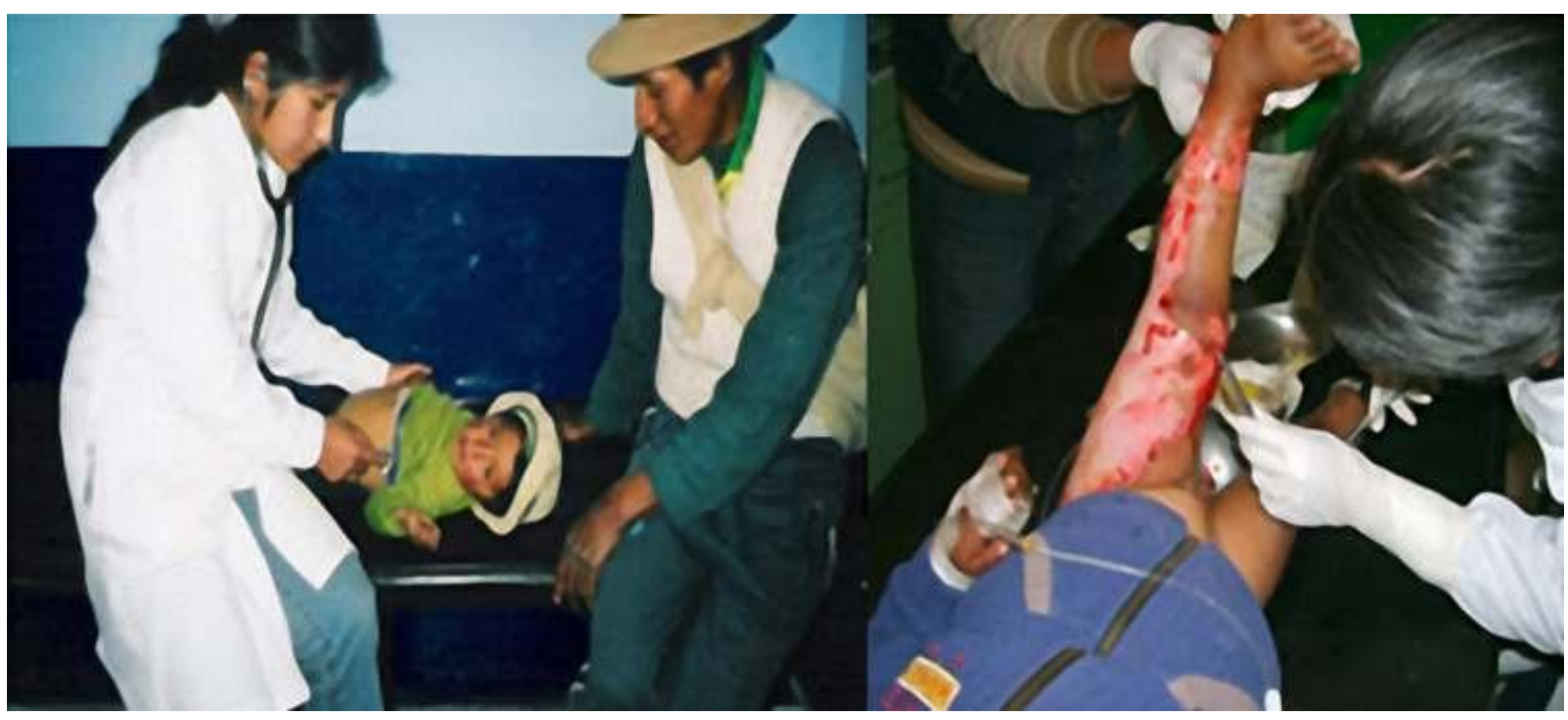

1 Doctor en Medicina de la Facultad de Medicina de la Universidad Nacional Mayor de San Marcos. Profesor Principal de la Escuela Profesional de Medicina Humana de la Universidad Nacional de San Antonio Abad del Cusco. E-mail: Santiago.saco@unsaac.edu.pe 
incorporando la enseñanza teórica y práctica de la Medicina Preventiva, Social y de la Medicina Comunitaria, la interdisciplinariedad (colaboración intersectorial), así como el uso de los diversos servicios de salud, que permitan la incorporación del estudiante a los diferentes niveles de atención, y el aprendizaje en el trabajo. (3)

De acuerdo a los lineamientos antes mencionados, la Facultad de Medicina Humana de la Universidad Nacional de San Antonio Abad del Cusco implementa actividades comunitarias en la formación médica. Los estudiantes desde los primeros años hacen prácticas en los centros de salud urbano-marginales y rurales del Cusco; incluyéndose estas actividades en los cursos clínicos (Medicina, Pediatría, Gineco-obstetricia y Cirugía) y no solo en los servicios hospitalarios del tercer nivel. En el penúltimo año los estudiantes realizan un "Internado Rural", durante 3 meses, en un establecimiento de salud del área rural, incorporándose el interno en el trabajo del equipo de salud del establecimiento . Los estudiantes quedan bajo la tutoría de los médicos del establecimiento y son supervisados mensualmente por los docentes de la Facultad.

La Universidad Nacional de San Antonio Abad del Cusco, gestionó y logró establecer una serie de convenios con el Ministerio de Salud a nivel nacional y regional para garantizar la ejecución de la Integración Docente - Asistencial.

La presencia de los estudiantes y docentes constituye un elemento dinamizador de las actividades de salud, sobre todo de la promoción y prevención de la salud, lo cual contribuye a mejorar la calidad de los servicios de salud.

La Facultad de Medicina Humana de la Universidad Nacional de San Antonio Abad del Cusco viene desarrollando este programa desde 1980, hasta la actualidad.

Fotografia 2. Control de crecimiento y desarrollo de la niña y el niño menor de cinco años. 


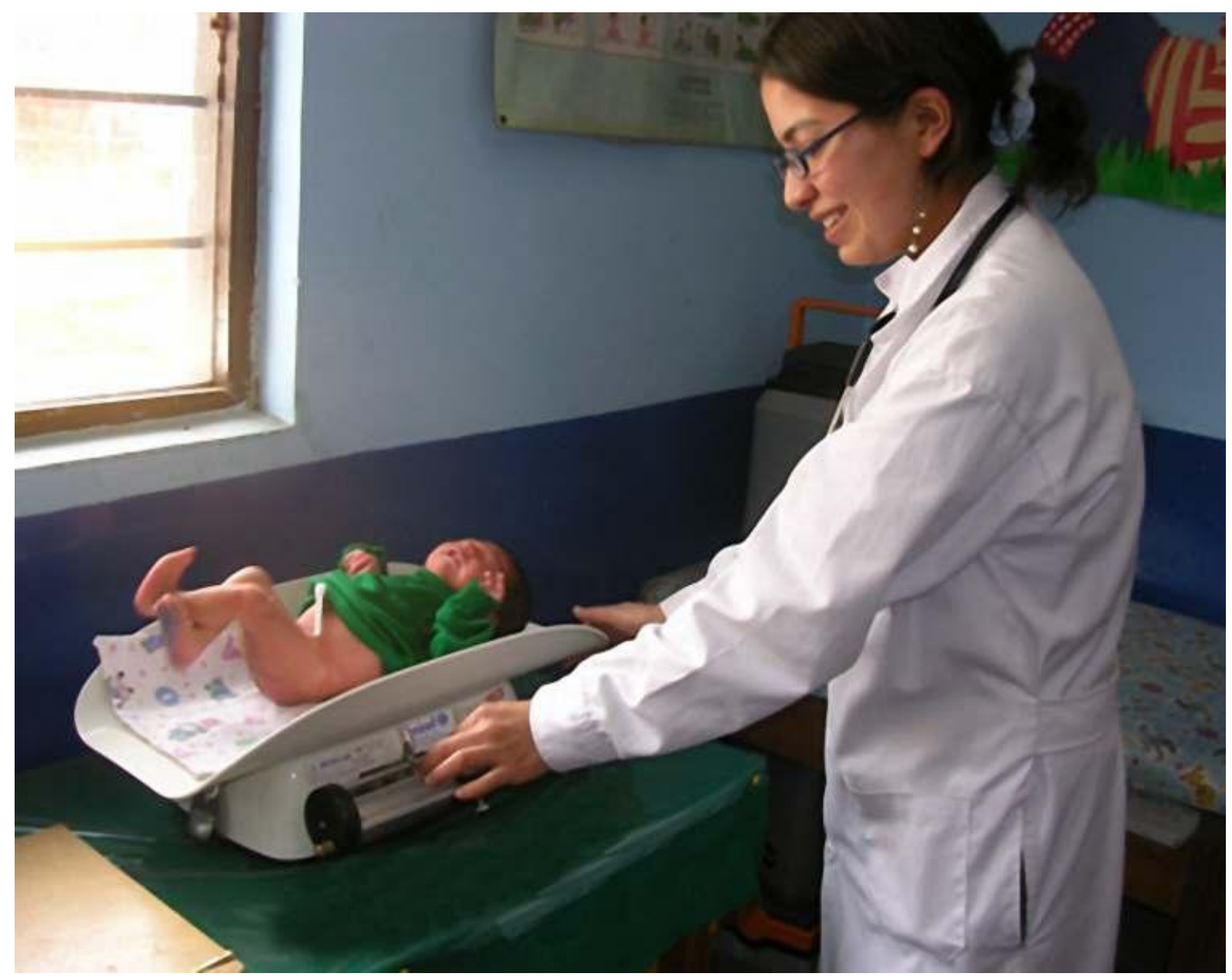


Fotografía 3. Atención de consulta externa.

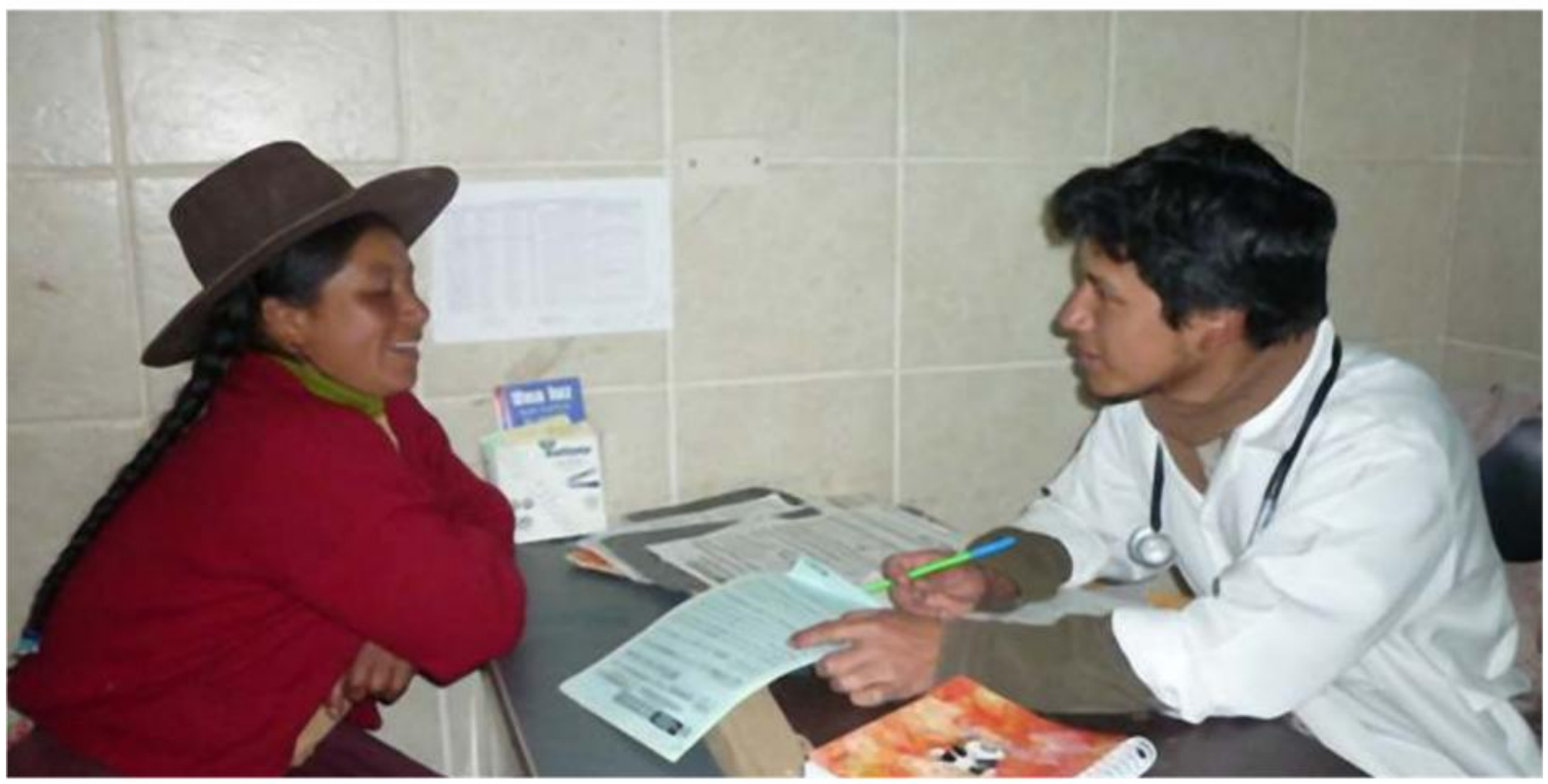

Fotografía 4. Campaña de vacunación.

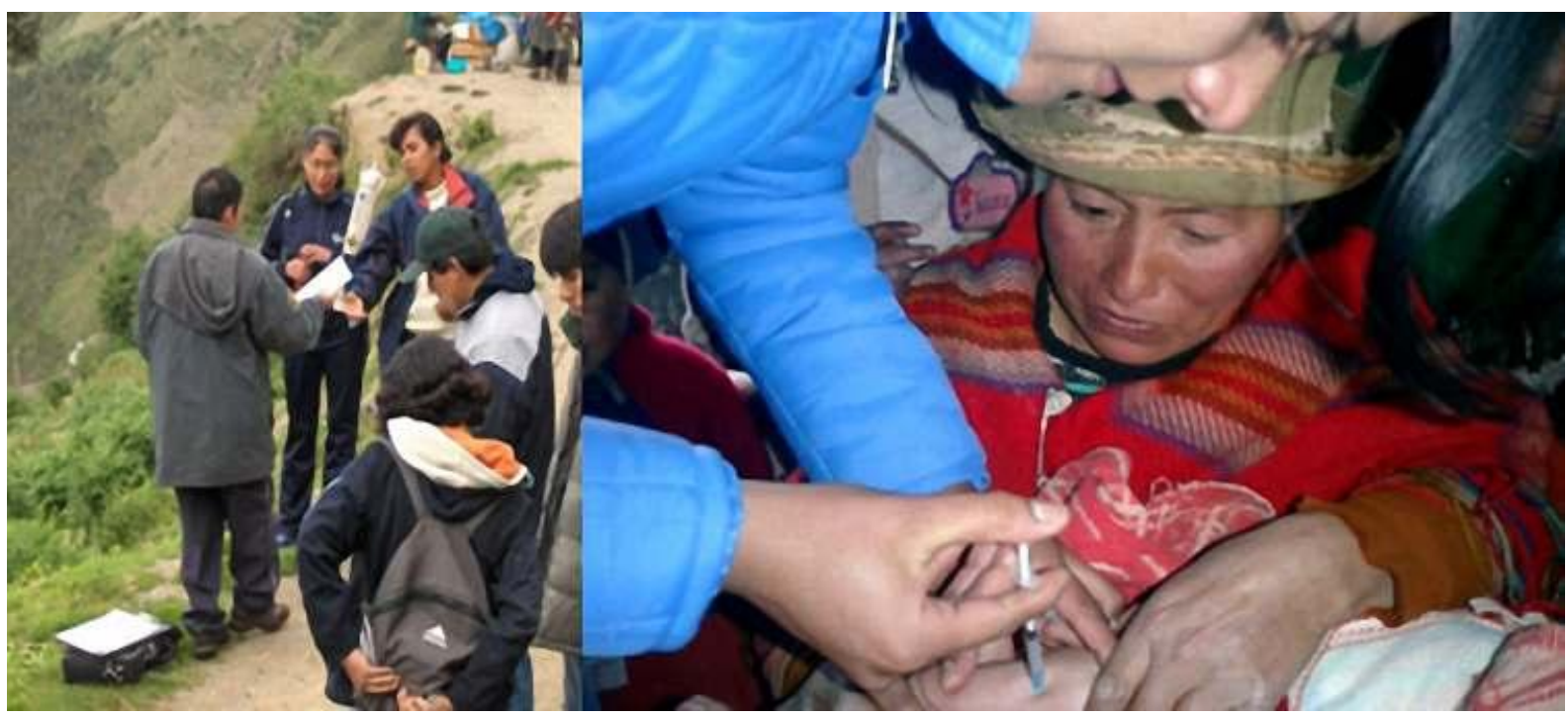

Un apoyo importante para el desarrollo de este programa fue de la Cooperación Técnica Alemana (GTZ) que en 10 años de apoyo, capacitó a los docentes, equipó a la facultad y construyó 06 módulos docentes en los Centros de Salud de Huancarani, Paucartambo, Pilcopata, Quiquijana, Ccatcca y Ocongate y dotó de 04 unidades móviles, incluyendo el apoyo de los médicos asesores alemanes Dra. Angelika Schrettenbrunner y Dr. Wolf Wagner.

La Facultad también recibió apoyo del Ministerio de Economía y Finanzas, y de la Cooperación Italiana.
Desde 1997, estudiantes de medicina de la Facultad de Medicina de la Universidad de Louvain (Bélgica) participan en el internado rural, realizando las mismas actividades de los estudiantes cusqueños, gracias a una iniciativa de la Dra. Myriam Melengreau, profesora de esa casa de estudios.

Presentamos una galería de fotografías tomadas por los estudiantes de medicina que realizaron el Internado Rural. Las fotografías forman parte de sus informes que presentan al finalizar su práctica rural.

Los alumnos encuestados consideraron que el internado rural les pareció el curso 


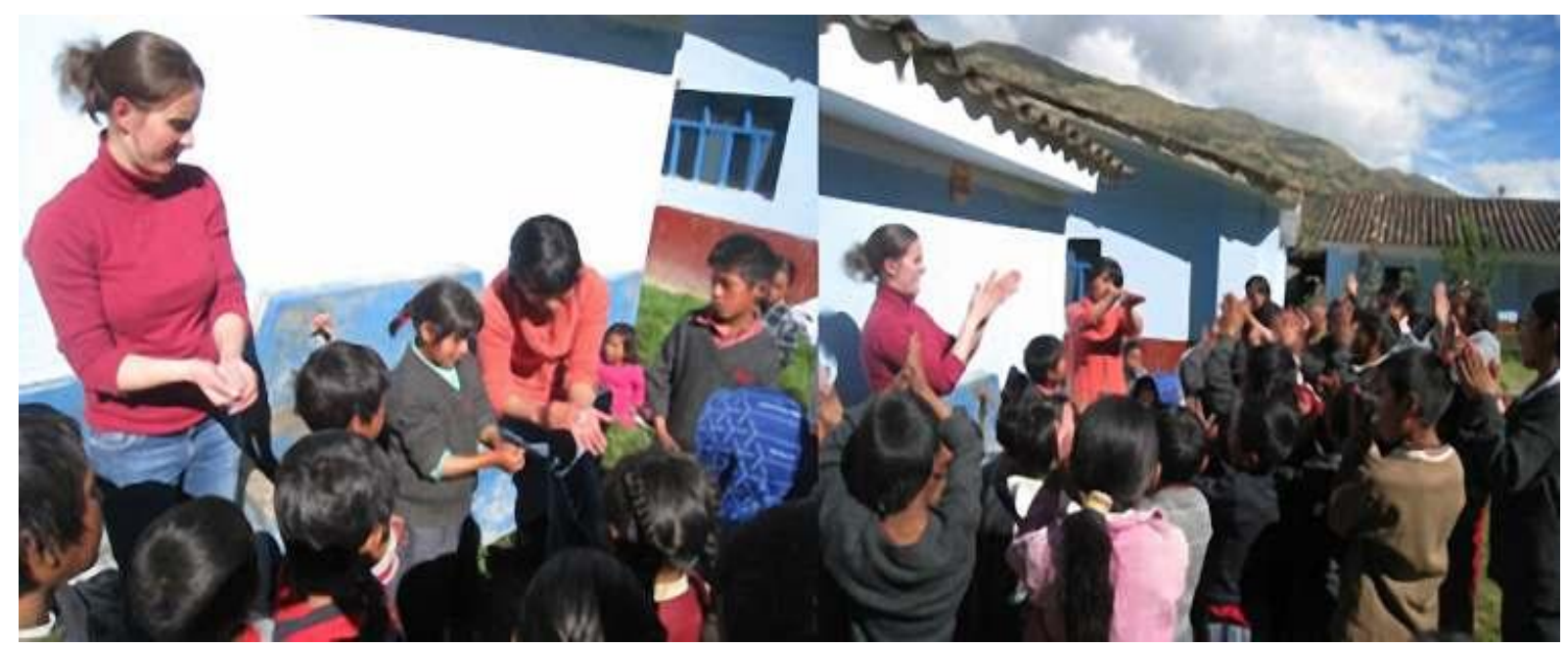

más interesante de la formación médica, siendo esto un logro importante de la experiencia. Se explicaría porque es un curso eminentemente práctico, donde los alumnos ponen en práctica los conocimientos aprendidos durante los 6 años de estudios en la Facultad y en los servicios de salud, donde ven otra realidad tanto de la comunidad como de los servicios de salud, trabajan en comunidades organizadas, y tienen la oportunidad de tomar decisiones, es que lo hace atractivo.

En la apreciación general del internado rural, el $81 \%$ de los estudiantes encuestados valora al Internado Rural como bueno y excelente. Esto es un logro muy importante y estimulante para el trabajo que venimos realizando.

En resumen se puede decir que el Internado Rural aporta muchas ventajas en la formación integral del médico, desde competencias cognitivas, procedimentales y actitudinales como:

1. Conocer la realidad socio cultural, económica y de salud de comunidades andinas y amazónicas de extrema pobreza. Esta realidad representa el futuro primer escenario de trabajo del médico joven. Conociéndola, le permite contribuir a mejorar sus condiciones de salud y de vida.

2. Conocer la dinámica de trabajo y la gestión de un establecimiento del primer nivel de atención de salud, y apoyar en el desarrollo de sus actividades.

3. Conocer la patología de estas zonas, aprender y aplicar las estrategias de salud del Ministerio de Salud para la promoción, prevención, curación y rehabilitación.

4. Conocer e interpretar los factores determinantes del proceso salud-enfermedad de estas zonas.

5. Conocer y valorar los patrones culturales (idioma, costumbres), la cosmovisión de salud-enfermedad y las prácticas médicas con las que la comunidad busca resolver sus problemas de salud.

6. Conocer la dinámica de la organización comunal y de su participación en la solución de sus problemas de salud.

7. Realizar investigaciones sobre problemas prioritarios de salud.

8. Conocer y aplicar las diversas metodologías de capacitació $n$ de poblaciones.

9. Realizar actividades de medicina legal.

10. Desarrollar actividades de saneamiento básico.

11. Tener una verdadera formación médica integral. 
Fotografía 6. Enseñando el cepillado de dientes en escuelas.

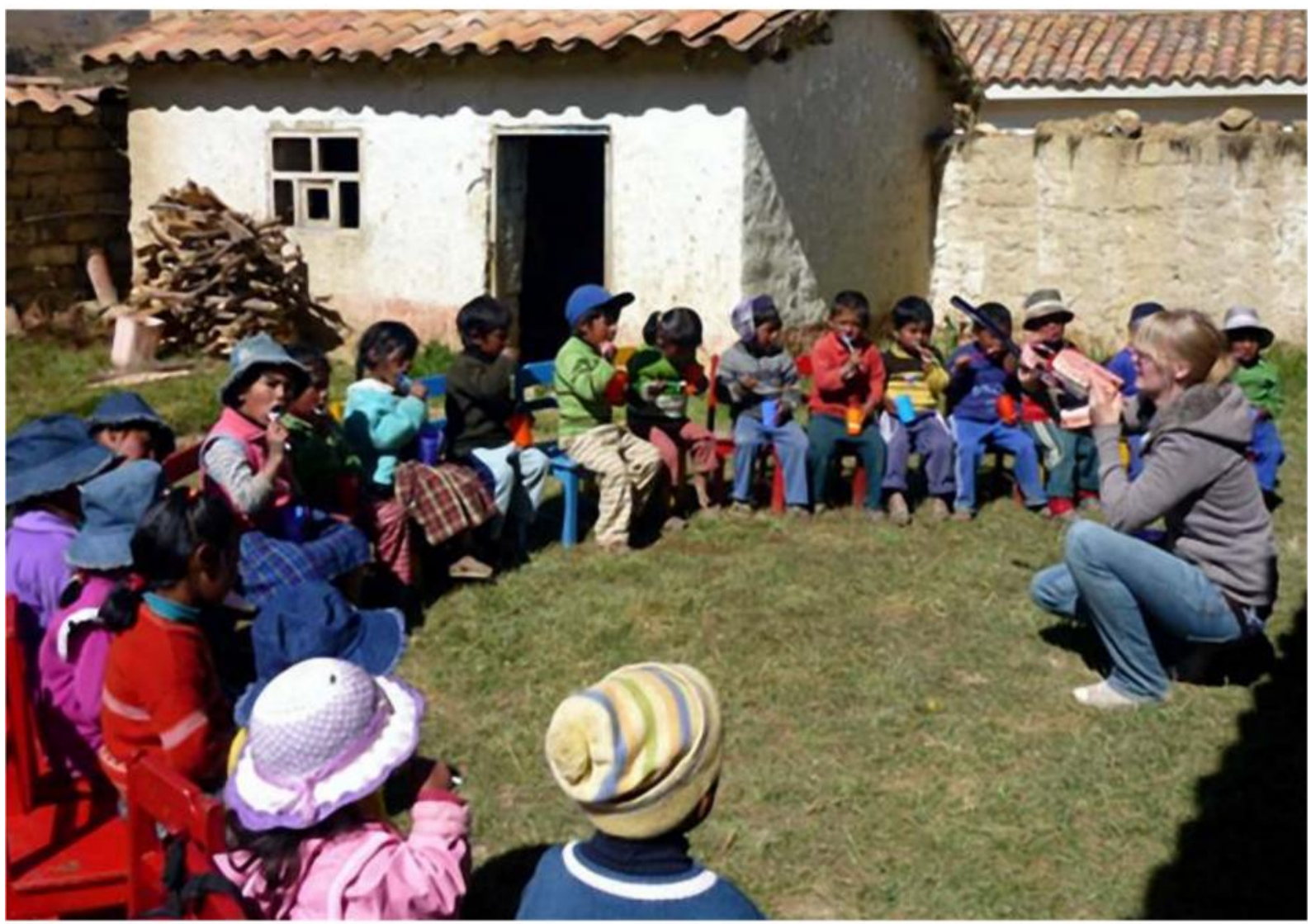

Fotografia 7. Concurso de casas limpias y ordenadas.

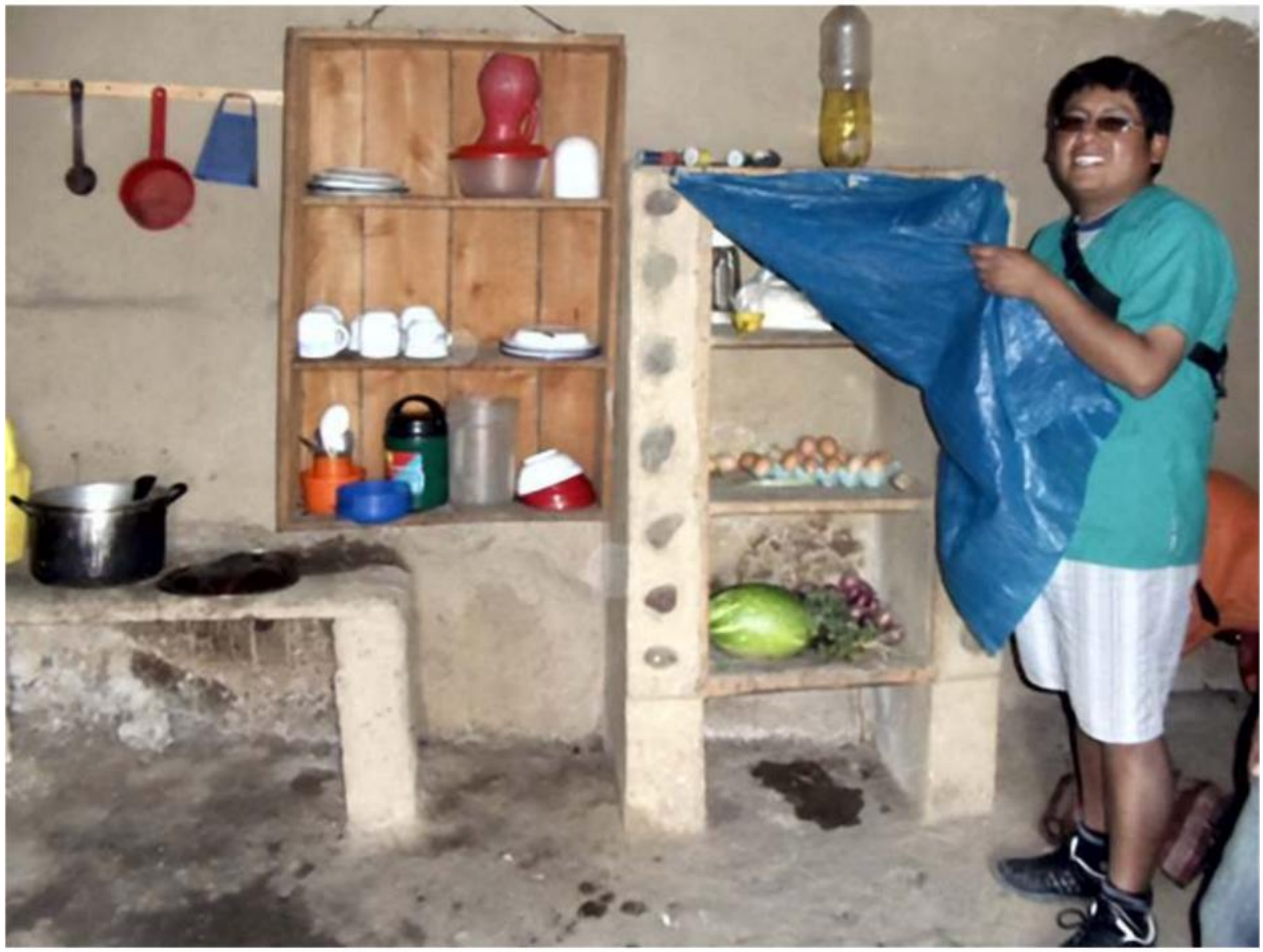


Fotografia 8. Construcción de letrinas.

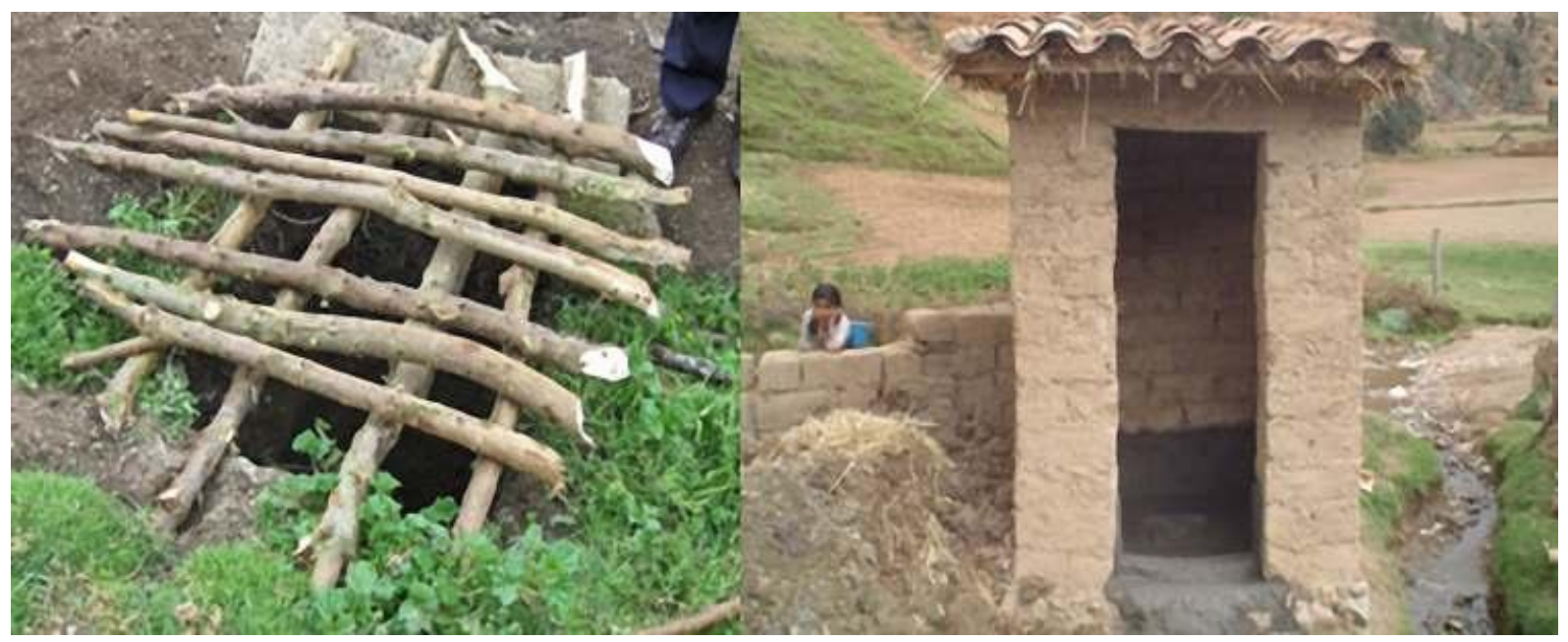

Fotografia 9. Centro de Salud Paucartambo.

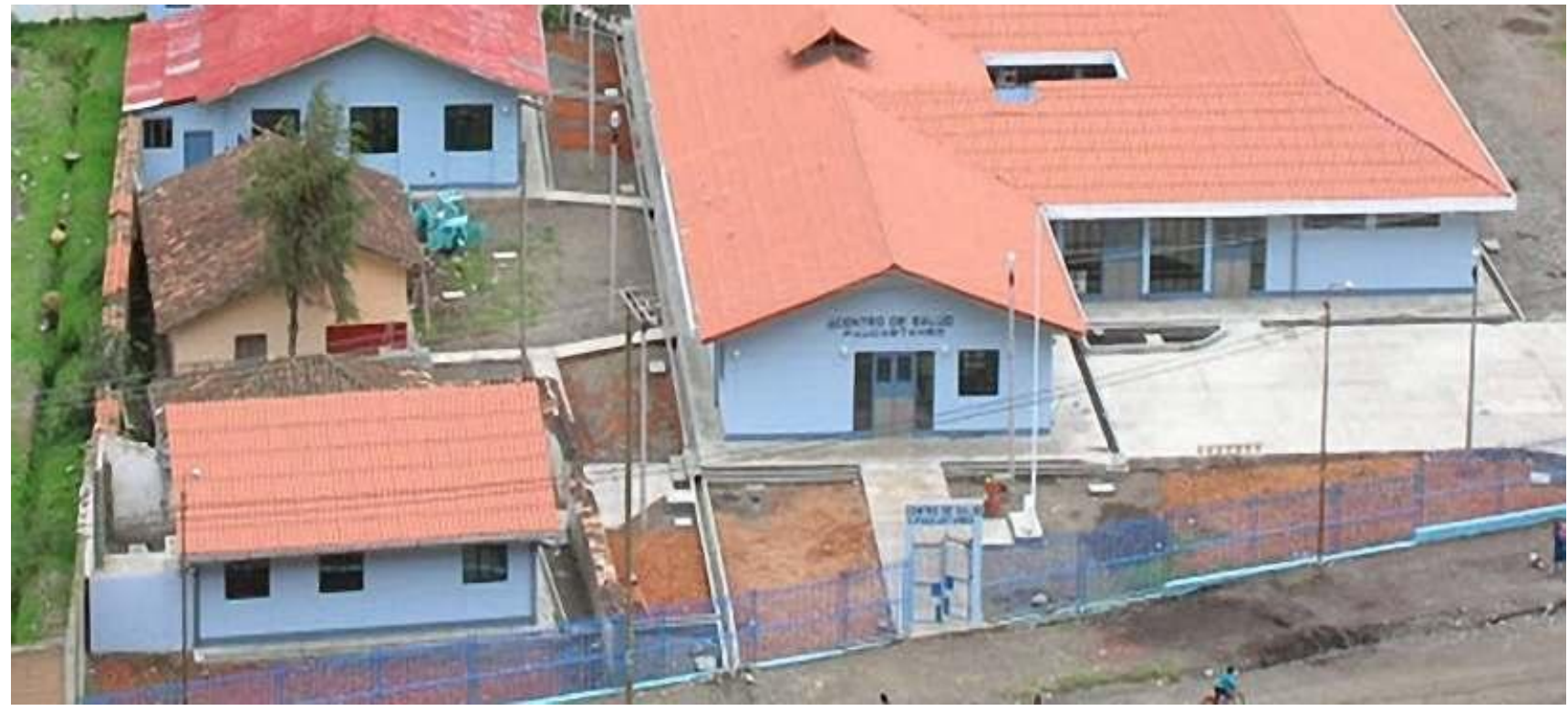

Fotografia 10. Interna belga actuando junto con los internos cusqueños en un sociodrama.

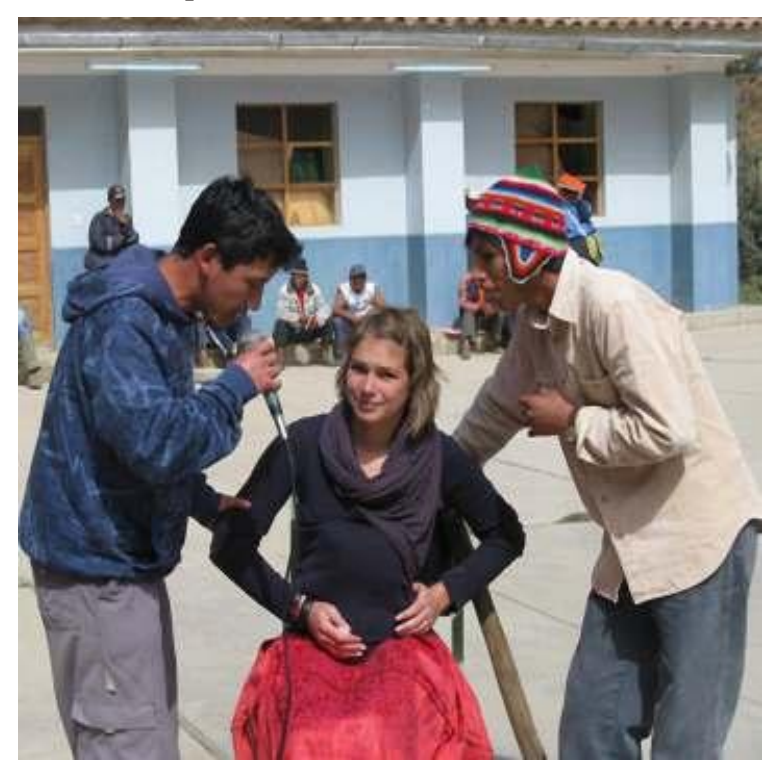

\section{TESTIMONIOS}

Presentamos los testimonios del interno rural, hechos por los internos:

1. Interno Rural Sandro Villafuerte Dueñas:

"Todos estos años de esfuerzo constante, de noches de desvelo, de andar interminable por caminos inciertos. Es en este periodo de mi vida es que encuentro el pedacito de vocación que me faltaba, la chispa para continuar con la llamada de mi aprendizaje y mi futuro... Servir. El Internado Rural prepara al futuro médico de manera inigualable para su desempeño posterior y además revive lo 
Fotografía 11. Campaña de Vacunación en Pilcopata.

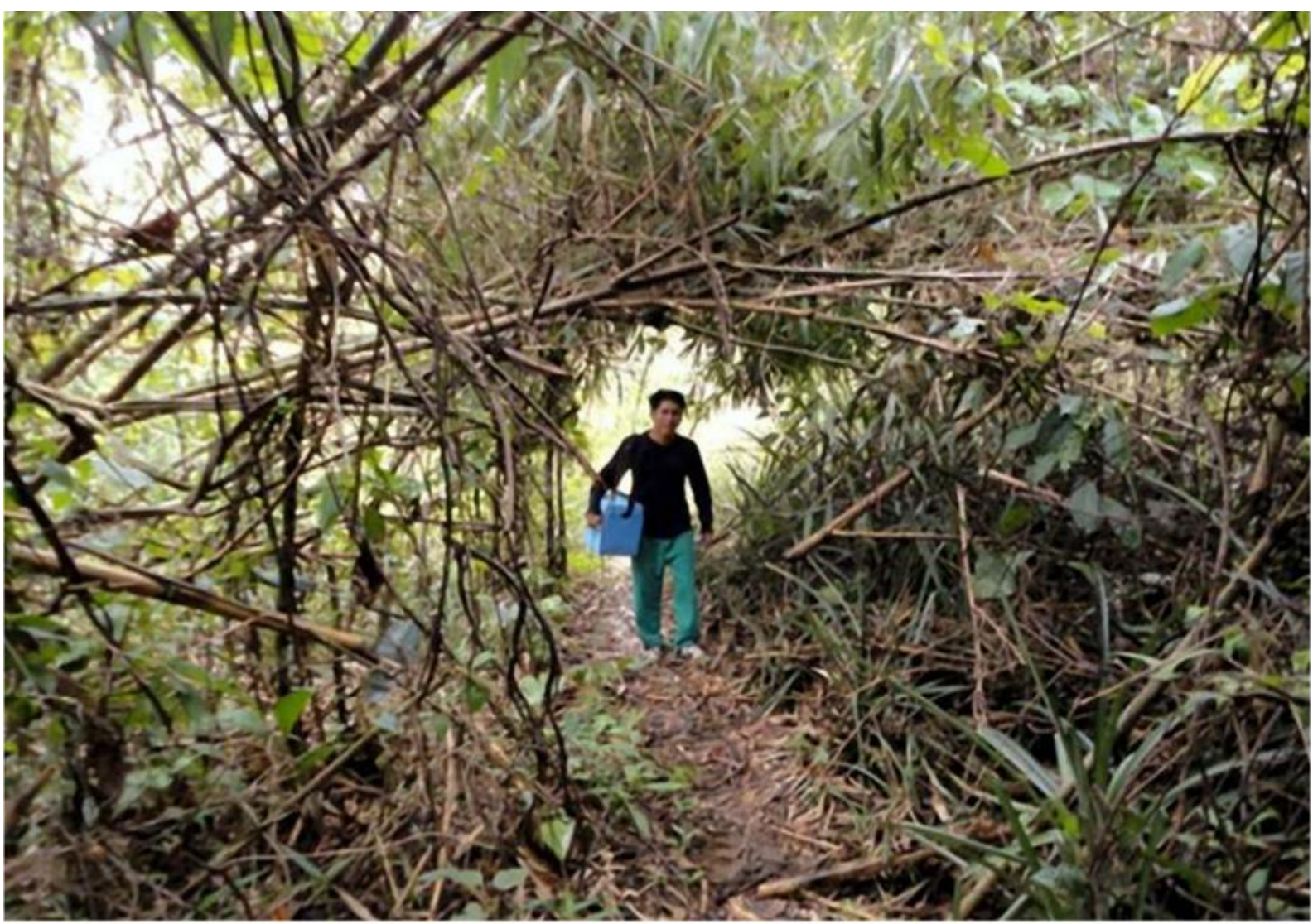

Fotografía 12. Atención Comunitaria en Queros Paucartambo.

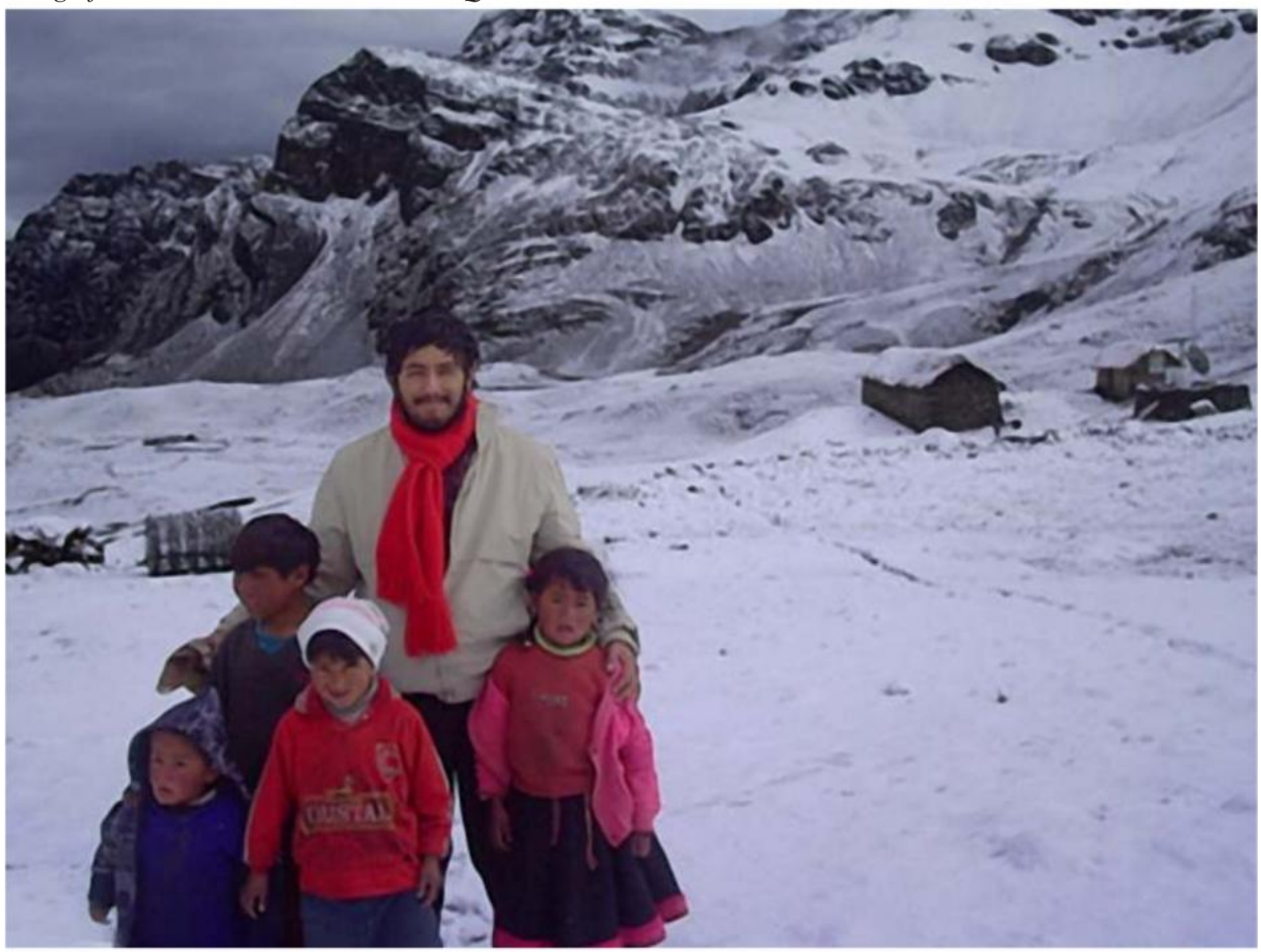


que en muchas ocasiones olvidamos... lo humano que debemos ser. Para mí ha sido la experiencia más intensa en mi vida y ha colmado las expectativas que acumulé durante años de estudio, ahora ya lejos de las aulas y en el Internado Rural siento que mi esfuerzo no fue en vano. Agradezco a mis docentes y a todo aquel que se preocupó en enseñarme no solo de medicina."

2. Internos Marcela Mar Meza y Víctor Raúl Valdivia Calderón: "A lo largo de los 7 años transcurridos en la Facultad de Medicina de la Universidad Nacional de San Antonio Abad del Cusco, hemos sido participes de muchas experiencias que nos han acercado a diferentes realidades humanas, las cuales nos han enriquecido como profesionales, y principalmente como seres humanos. Pero la experiencia obtenida durante la realización del Internado Rural, es una experiencia nueva, que enfrenta nuestro nivel profesional y humano, con la realidad totalmente diferente, nos permite ser conocedores de todas las carencias que puede sentir el ser humano dentro de las comunidades campesinas; en una sociedad cercana al segundo milenio y que se jacta de estar explorando otros planetas. El haber convivido con personas de patrones sociales, culturales y económicos diferentes, nos hacen ver que en salud hay mucho trabajo que hacer y nuestros niveles preventivos-promocionales aún no están logrando las coberturas necesarias, para lograr cambios en los hábitos $\mathrm{y}$ costumbres necesarios en salud. Como futuros profesionales, cercanos a trabajar en estas realidades, nos compromete a poner el mejor desempeño y hace que nuestro tiempo de servicio rural médico, sirva para abocar nuestro trabajo en contribuir a cambiar esta realidad."
3. Interna Yovana Vera: "El Internado Rural es una experiencia real, donde no solo se ponen en práctica los conocimientos médicos, sino en la parte humanística, que todos tenemos que palpar la cruda realidad de las comunidades campesinas, caracterizadas por una pobreza extrema que no cubre sus necesidades mínimas. Nosotros los Internos de Medicina y todos los médicos tenemos grandes retos no solo con la comunidad sin con nosotros mismos, debem os vencer muchos perjuicios infundados, debemos dejar de lado el egoísmo, los celos profesionales y trabajar juntos de la mano."

4. Interna Yamith Soto Cortez: "El Internado Rural es una etapa que nos brinda la oportunidad de captar en forma directa la realidad de nuestra sociedad, la que muchos creemos conocerla, sin embargo este concepto cambia cuando a dar la vuelta de un cerro, uno se encuentra con un mundo diferente, otra cultura, otras formas de pensa $r$, otras costumbres, formas diferentes de ver la vida y donde existen problemas no percibidos por nuestra visión miope."

\section{REFERENCIAS}

1. Vidal, C., Quiñones J. (1986) "Integración Docente Asistencial”, Organización Panamericana de la Salud Educación Médica y Salud, Vol. $20 \mathrm{~N}^{\mathrm{o}} 1$. Washington, Estados Unidos de América.

2. Organización Mundial de la Salud, Fondo de las Naciones Unidas para la infancia UNICEF, Alma-Ata: "Atención Primaria de Salud". Informe de la Conferencia Internacional sobre Atención Primaria de Salud, Alma-Ata, Unión de Repúblicas Socialistas Soviéticas, 6-12 septiembre 1978. Washington Estados Unidos de América.

3. Covarrubias, H. et al. 1977 "Proyecto para la creación del Programa Académico de Medicina Humana de la Universidad Nacional de San Antonio Abad del Cusco." (mimeografiado). Cusco.

4. Universidad Nacional de San Antonio Abad del Cusco - Facultad de Medicina Humana. (1987)

"Integración Docente Asistencial en Atención Primaria" (mimeografiado). Cusco. 


\section{ASPECTOS GENERALES}

La Revista El Antoniano tiene por finalidad la publicación de artículos científicos y culturales que contribuyan al desarrollo a nivel local, nacional e internacional, en los diferentes ámbitos científicos. La revista publicará preferentemente artículos de investigación inéditos, pero también se considerarán trabajos de otros tipos, como los estudios de casos y revisiones teóricas (libros y artículos), temas de revisión, revisión de revistas, cartas al editor (ver anexo 1) y galería fotográfica, que luego de haber sido aprobados por el comité científico de la revista serán considerados para su posible publicación.

Todos los trabajos enviados deben ser inéditos, dicho de otra forma, no haber sido publicados en ningún otro medio, local, nacional ni internacional. Una vez enviado el trabajo el autor asume que este será destinado para su valoración y difusión, si así fuera el caso.

Una vez publicado el artículo científico, el autor o autores transfieren los derechos de copyright a la Revista El Antoniano de la Universidad Nacional de San Antonio Abad del Cusco.

El comité científico se reserva el derecho de publicación del artículo en el número de la revista que considere oportuno. De la misma forma, el comité científico entiende que las opiniones y puntos de vista vertidos por el autor o autores son de su entera responsabilidad.

\section{PRIMERO: SOBRE EL ENVÍO}

Los trabajos podrán enviarse de tres maneras:

1. ENVIANDO CORREO ELECTRÓNICO dirigido al Director de la Revista El Antoniano de la Universidad Nacional de San Antonio Abad del Cusco, adjuntando los archivos electrónicos a la siguiente dirección elantoniano@unsaac.edu.pe.

2. ENTREGADO EN LAS OFICINAS de la Unidad de Responsabilidad Social de la Universidad Nacional de San Antonio Abad del Cusco, Av. de la Cultura 733, Cusco, en sobre cerrado conteniendo la impresión en un fólder, acompañado de un disco compacto conteniendo los archivos electrónicos, dirigido al Director de la revista El Antoniano.
3. POR CORREO REGULAR conteniendo la impresión en un folder, acompañado de un disco compacto conteniendo los archivos electrónicos, con destinatario a: Director de la Revista El Antoniano, de la Universidad Nacional de San Antonio Abad del Cusco, Unidad de Responsabilidad Social, Av. Cultura 733, Cusco Perú.

\section{SEGUNDO: LA PRESENTACIÓN}

El trabajo debe estar escrito en lengua española, tener una extensión máxima de 25 carillas a doble espacio, equivalente a 50.000 caracteres. Todos los márgenes deberán medir $2.4 \mathrm{~cm}$, letra Arial tamaño 12 puntos.

Las partes de presentación del trabajo debe considerar el siguiente orden:

1. La primera página se estructurará mediante el siguiente orden:

a) Título del trabajo,

b) Primer apellido y nombre y del autor o autores y grado académico y/o título profesional,

c) Nombre y dirección de la institución,

d) Dirección domiciliaria, dirección de correo electrónico y número de teléfono del autor responsable para la correspondencia,

e) Agradecimientos (si los hubiera), y

f) Título abreviado del trabajo.

2. La segunda página deberá contener:

a) Título del trabajo abreviado, entre 10 a 15 palabras.

b) Resumen con un máximo de 200 palabras. El resumen de los trabajos originales será estructurado bajo la siguiente organización:

-Introducción.

-Métodos

—Resultados

- Conclusiones

c) Palabras clave. Entre 3 y 8 palabras.

3. La tercera página deberá contener la traducción y redacción en inglés del contenido de la página segunda: title, abstract y keywords. 
4. Las comunicaciones cortas deben tener: resumen, introducción breve, texto de la comunicación, discusión y referencias.

El total de páginas, incluyendo sus referencias (máximo 10) no debe ser mayor de 5 paginas, escritas a doble espacio. El resumen no tendrá más de 100 palabras.

5. Las cartas al Director, estarán compuestas por el texto de la carta y las referencias: no debe tener más de dos páginas.

6. Las galerías fotográficas: Se refiere al envío de fotografias de interés sobre un tema, acompañado de un breve resumen del tema y una explicación de las fotografias. El comité editorial de la revista se reserva el derecho de limitar el número de ilustraciones.

Las páginas siguientes se dedicarán al texto del trabajo, que conviene esté dividido en secciones delimitadas (en los trabajos de investigación: introducción, método, resultados, discusión, conclusiones y referencias bibliográficas).

Las tablas y las figuras se incluirán después de las referencias (cada tabla y cada figura debe ir en página aparte), irán numeradas correlativamente según su aparición en el texto, debiendo especificarse su posición en este (indicar la ubicación aproximada dentro del texto). Las tablas que se incluyan, deben seguir los criterios de claridad y sencillez. Las figuras no deben de ser tridimensionales a menos que exija el diseño estadístico. Debe ponerse extremo cuidado en no repetir, mediante las tablas y figuras la información (datos) ya contenidos en el texto, así como en no solapar información entre tablas y figuras.

Siempre que sea posible, se aconseja utilizar tablas en lugar de figuras.

El estilo del trabajo y la forma de nombrar las citas y referencias, deberá ajustarse a las normas internacionales de la American Psychological Association (APA), las que se podrán consultarse en el Manual de Estilo de Publicación de la American Psychological Association 5ta. edic. 2002, adaptado para el español por la Editorial El Manual Moderno, así mismo, podrá considerar en el estilo de Vancouver, que podrá ser consultado en: http://web.ua.es/es/eurle/documentos/trabajo-defin-de-grado/estilo-vancouver.pdf; finalmente si se tratase del estilo Harvard, puede ser consultado en: https://rua.ua.es/dspace/bitstream/10045/45402 /1/Estilo-Harvard.pdf
Cada referencia tiene que estar completa: apellido e iniciales del nombre de cada autor titulo del artículo; nombre entero de la revista con el número del volumen y páginas o ciudad de publicación del libro, nombre de la editorial y fecha de la publicación.

Sin embargo, deben de considerarse las siguientes normas mínimas:

EN RELACIÓN A LAS REFERENCIAS, el autor debe asegurarse de que, cada una de las referencias citadas indefectiblemente, deben figurar en el apartado de referencias que estarán en orden alfabético y se ajustarán al formato de libro, artículos de revistas científicas, capítulos de libro y referencia de Internet, y artículo de dominio público, revista electrónica o base de datos libre (monografía), de la manera siguiente:

A. LIBRO: Apellidos, iniciales del o los nombres. Año de publicación entre paréntesis. Título de la obra en cursivas. Lugar de publicación: Nombre de la editorial. Ejemplo: Toro, I. y Parra, R. (1997). Fundamentos epistemológicos de la investigación y de la metodología de la investigación. Medellin: Fondo Editorial Universidad EAFIT.

B. ARTÍCULO DE REVISTA CIENTÍFICA: Apellido/s, inicial/es del o de los nombres. Año de publicación entre paréntesis. Título del artículo. Nombre completo de la revista en cursivas, número del tomo en cursivas, y, número del volumen en cursivas, números completos de la primera y última página del artículo. Por ejemplo: Montero, I. y León, O.G. (2007). A guide for naming research studies in Psychology. International Journal of Clinical and Health Psychology, 7, 847-862.

C. CAPÍTULO DE LIBRO: Apellido/s, iniciales del o de los nombres. Año de publicación entre paréntesis. Título del capítulo, iniciales y apellido/s del editor o recopilador. Título del libro en cursivas. Números de primera y última páginas del capítulo. Lugar de publicación: Nombre de la editorial. Ejemplo: Arnau, J. (1995). Metodología de la investigación psicológica. En M. T. Anguera, J. Arnau, M. Ato, R. Martínez, J. Pascual y G. Vallejo (eds.): Métodos de investigación en psicología, p. 23-43. Madrid: Síntesis.

D. REFERENCIA DE INTERNET: Cuando se emplee este tipo de referencias debe incluirse la 
totalidad de la dirección URL. Si las direcciones incluyen nombres de autor, se citarán en el texto de forma similar al resto de referencias y se incluirán, igualmente, en la sección de referencias. Si no incluyen autor, se referirán el organismo o asociación, que se incluirá siguiendo el orden correlativo según su aparición en el texto. Ejemplo: Organización Mundial de la Salud (2002). Informe sobre la salud en el mundo 2002 - Reducir los riesgos y promover una vida sana. Recuperado el 10 de junio del 2009 de http://www.who.int/whr/2002/en/whr02_es.pdf.

\section{E. ARTÍCULO DE DOMINIO PÚBLICO, REVISTA} ELECTRÓNICA O BASE DE DATOS LIBRES (MONOGRAFÍAS). Cuando se emplee este tipo de referencias debe incluirse la totalidad de la dirección URL. $\mathrm{Si}$ las direcciones incluyen nombres de autor, se citarán en el texto de forma similar al resto de referencias, indicando el mes y día a lado del año, que se incluirán, igualmente, en la sección de referencias, siguiendo el orden correlativo según su aparición en el texto. Ejemplo: Lodewijkx, H. F. M. (2001, Mayo 23). Individual-group continuity in cooperation and competition under varying communication conditions. Current Issues in Social Psychology, 6(12), 166-182. Extraído el 14 de septiembre de 2001 desde http://www.uiowa.edu/ grpproc/ crisp/crisp.6.12.htm.

TERCERO: EL PROCESO DE REVISIÓN

Una vez recibido el comunicado de recepción del trabajo, el cual será enviado por el comité editorial de la revista (vía correo electrónico) al autor principal, se enviará al consejo editorial de la revista, para su evaluación y juzgamiento sobre la conveniencia de su publicación.
En el caso de encontrar observaciones, el comité encargado comunicará al autor, para que en un plazo de 15 días haga las correcciones sugeridas y vuelva a remitir una nueva versión del trabajo, pasado este plazo se desestimará su publicación.

Una vez aceptado, el texto final se enviará al autor principal para su aprobación antes de mandarlo a la composición topográfica, el comité editorial se reserva el derecho de no publicar un manuscrito si los autores no dan su visto bueno al texto editado.

El método de revisión empleado es de doble ciego (anonimato de autor y evaluadores), siendo un miembro del consejo editorial el encargado de establecer contacto entre ambos.

\section{CUARTO: LA PUBLICACIÓN}

Una vez publicado el artículo, el autor recibirá un ejemplar del correspondiente número de la revista El Antoniano.

\section{REFERENCIAS}

American Psychological Association: APA Style: Recuperado el 07 de noviembre del 2009 de: http://www.apastyle.org/ elecref.html

Graham, S. (2006). Strategy instruction and the teaching of writing: A meta-analysis. En C.A. MacArthur, S. Graham y J. Fitzgerald (Eds.), Handbook of writing research, p. 187-207. New York: Guilford Press.

Hartley, J., Pennebaker, J. W. y Fox, C. (2003). Abstracts, introductions and discussions: How far do they differ in style? Scientometrics, 57(3), 389-98.

Murray, R. \& Moore, S. (2006). The handbook of academic writing: A fresh approach. Maidenhead: Open University Press.

Instituto Nacional de Salud, Revista Peruana de Medicina Experimental y Salud Publica (2010) Lima.

Organización Panamericana de Salud, Revista Panamericana de Salud Publica, Washington 2000.

Anexo: Estructura de acuerdo al tipo de documento.

\begin{tabular}{lcccc}
\hline & Artículos originales & Reportes de caso & Artículos de revisión & Carta al editor \\
\hline Título del articulo & $\times$ & $\times$ & $\times$ & $\times$ \\
\hline Resumen & $\times$ & $\times$ & $\times$ & \\
\hline Abstract & $\times$ & $\times$ & $\times$ & \\
\hline Introducción & $\times$ & $\times$ & Texto al editor \\
\hline Contenido & $\begin{array}{c}\text { Materiales } \\
\text { y métodos }\end{array}$ & $\begin{array}{c}\text { Presentación del caso (anexo acompanar } \\
\text { el consentimiento informado del paciente) }\end{array}$ & $\begin{array}{c}\text { Tópicos del } \\
\text { tema de revisión }\end{array}$ \\
\hline Resultados & $\times$ & $\times$ & & \\
\hline Discusión & $\times$ & $\times$ & & \\
\hline Conclusiones & $\times$ & $\times$ & $\times$ \\
\hline Referencias & $\times$ & $\times$ & \\
\hline
\end{tabular}


